\title{
H. pylori infection and gastric cancer in Bangladesh: a case-control study: erratum
}

In this article that appeared in the November 2017 issue, there was a mistake in the author list. The author list should appear as follows:

Mohammed Abu Kawsar Sarker, FCPS, MS, Md. Jahangir Kabir, FCPS, MS, MRCS, A.K.M. Minhaj uddin Bhuyian, FCPS, MS, MRCS, Md. Shahjadul Alam, FCPS, MS, Fazle Rabbi Chowdhury, FCPS, MSC, M. Abdul Ahad, FCPS, MD, Md. Anisur Rahman, FCPS, M. Mizanur Rahman, FCPS

\section{Reference}

[1] Sarker MAK, Kabir MJ, Bhuyian AKMM, et al. H. pylori infection and gastric cancer in Bangladesh: a case-control study. IJS Oncol 2017;2:e44. Doi: 10.1097/IJ9.0000000000000044. 